\title{
Influence of Various Process Variables and Formulation Excipients on the Engineering of Sertaconazole Solid Lipid Nanoparticles
}

\author{
Anagha Baviskar*1 ${ }^{1}$, Shivanand Hiremath ${ }^{2}$, N. Devanna ${ }^{3}$, Manoj akul ${ }^{4}$ \\ ${ }^{1}$ Jawaharlal Nehru Technological University Anantapur, Andhra Pradesh, India. \\ ${ }^{2}$ Pravara rural College of pharmacy, Loni, Maharashtra, India. \\ ${ }^{3}$ Jawaharlal Nehru Technological University Anantapur, College of Engineering, Kalykiri- \\ 517234, Chitoor, Andhra Pradesh, India. \\ ${ }^{4}$ Research and Development, Glenmark Pharmaceuticals LTD, Nashik, Maharashtra, India.
}

\begin{abstract}
The present research work focuses on the study of influence of various ingredients, concentration of ingredients and process parameters on the formulation of Solid lipid nanoparticles (SLN). The sertaconazole loaded solid lipid nanoparticles (SERT-SLN) were prepared by simple, easily reproducible and novel modified solvent emulsification method. Lipid and surfactant solubilizing potential was determined by solubility study. To improve the properties of SLN some excipients were added like cosurfactant TPGS, organic solvent for nanodroplet structure in SLN and charge induction by stearyl amine. Further study was performed to determine the optimum concentration of components such as concentration of surfactant OLML, stearyl amine, TPGS and organic solvent. The process parameters like stirring rate and time, sonication time and cooling temperature also affect the formation of SLN. The formulations were evaluated for average particle size, polydispersity index (PDI) and zeta potential (ZP). The particle size, PDI and ZP of optimized formulation was observed to be 155.1 $\mathrm{nm}, 0.23$ and $9.76 \mathrm{mV}$ respectively. The TEM study confirms the nanometric size particles with particle size < $200 \mathrm{~nm}$. The DSC and XRD study reveals the presence of amorphous nature of SLN. The SERT-SLN formulation was stable at refrigerator and $25^{\circ} \mathrm{C}$. SERT-SLN showed biphasic release pattern initiated with burst and then sustained release.
\end{abstract}

Keywords: DMSO, Modified solvent emulsification technique, Natural lipid and surfactant, Solid lipid nanoparticles, TPGS.

\section{INTRODUCTION}

Solid lipid nanoparticles (SLN) were developed at the beginning of the 1990s as a colloidal carrier which was an alternative system to the existing traditional carriers like emulsions, liposomes, niosomes and polymeric nanoparticles. Nanoparticles made up of solid lipid have gained significant attention because of the drug immobility in solid lipid is more than any other carrier system. SLN have distinct advantages which include the possibility of incorporating drugs for controlled release, the low cytotoxicity due to its composition of physiological compounds, the possibility for loading both lipophilic and hydrophilic drugs into the solid matrix and for its likely large scale production. SLN also protect the drug or active pharmaceutical ingredients against the chemical, physical and environmental degradation. Due to these properties SLN have proved to be used for different routes of administration [1].The excipients used for the preparation of SLN are advantageous for the various drug delivery systems. The various types of solid lipids and surfactants have shown the significant solubilizing potential for lipophilic drug. The compatible nature of these excipients are more accepted and proved to be less allergic [2]. Among various route of administration, SLN has potentially explored as drug carrier for cosmetic and dermal products as SLN can favor drug penetration into the skin. The SLN have number of advantages like maintain a sustained release to avoid systemic absorption, act as a UV sunscreen system, reduce irritation and maintain the skin barrier homeostasis [3]. The dermal absorption of SLN can be influenced by number of factors such as location and skin conditions at the application site, physicochemical properties of the penetrating drug molecule, and physicochemical properties dosage form, others such as lipophilic-hydrophilic gradient, $\mathrm{pH}$ gradient and isoelectric point of formulation, formulation ingredients and their concentration i.e the presence of molecules such as solvents, surfactants, enhancers, liquid oil and others may alter or damage stratum corneum by different processes thus causing a potential increase in the absorption of all or selected ingredients of the applied formulation. Various parameters can affect the physicochemical properties of the SLN. Each formulation ingredient and its concentration, different formulation conditions, process and order of process can influence the formation of SLN.Sertaconazole nitrate (SERT), is an imidazole/triazole antifungal drug used to treat fungal skin infection. It is available as a topical cream as it has 
negligible bioavailability. Sertaconazole is a highly selective inhibitor of fungal cytochrome P-450 sterol C-14 $\alpha$-demethylation via the inhibition of the enzyme cytochrome P450 14 $\alpha$-demethylase. This enzyme converts lanosterol to ergosterol, and is required in fungal cell wall synthesis. Inhibition of cell wall synthesis results in increased cellular permeability causing leakage of cellular contents [4]. The drug is practically insoluble in water, thus supports a reason to formulate into lipoidal form. All these factors form a foundation to formulate a controlled release novel drug delivery system of sertaconazole nitrate.

In the present study, we have formulated the SERT loaded SLN using naturally derived lipid and surfactant and the influence of different formulation ingredients and process parameters that can significantly affect the properties of SLN. The use of naturally derived excipients like lipid (OLML) and surfactant (OLMS), D- $\alpha$ tocopheryl polyethylene glycol succinate (TPGS), stearyl amine (SA) and dimethyl sulfoxide (DMSO) would possibly improve the potential of SLN. By optimizing these parameters we can formulate a more stable, compatible, with maximum loading efficiency and enhanced skin targeting properties of SLN.

\subsection{Material}

\section{MATERIAL AND METHOD}

SERT was a kind gift by Cipla PVT Ltd, Mumbai, India. Lipid OLML and surfactant OLMS were generous gift from Chemhouse Marketing, Mumbai. D- $\alpha$ tocopheryl polyethylene glycol succinate (TPGS) was purchased from BASF India Ltd, Mumbai. Stearylamine was purchased from Sigma Aldrich chemicals PVT Ltd, Bangalore, India and other chemicals, such as stearic acid, glyceryl monostearate, tween 80, acetone, methanol, DMSO, poloxamer 188 and poloxamer 407 were of analytical grade and from Merck Chemical Company (Mumbai, India).

2.2 Pre formulation study

The formulation of solid lipid nanoparticles involves series of events which commences with selection of excipients along with the minimum effective concentration which gives the desirable outcome. Detailed study of this process gives insights about the influence of various parameters on the formulation of SLN. The preformulation study was started with selection of lipid and selection of surfactant.

2.2.1 Selection of lipid

The prime factors that determine drug release are the drug loading capacity and the pattern of drug loading. However, the study protocol is quite different from the equilibrium solubility study as for liquid solutions. Hence, a modified method is used to determine the maximum solubility of drug and lipids. For this study $50 \mathrm{mg}$ of SERT was taken in a test tube and lipid was gradually added in portions. The test tube was heated above the melting point of lipid and stirred continuously to confirm the mixing of drug and lipid. [5]. Before the solubility study the sertaconazole melting point was determined in lab to assure the stability of sertaconazole at respected melting point of lipid. The different solid lipids were screened such as stearic acid (SA), glyceryl monostearate (GMS), Myrj 49, compritol 888ATO, Lipid OLML and gelucire 44/14. The minimum amount of lipid required to solubilize $50 \mathrm{mg}$ of drug is observed and noted visually with the end point of clear or pale yellow molten solution of dissolved drug in lipid. The selection of lipid was based on the maximum solubilizing potential of solid lipid, stability of formulation and compatibility of lipid.

2.2.2 Selection of surfactant

The selection of surfactants, cosurfactants and other organic solvents was based on the equilibrium solubility of drug in the above solutions. The study includes the addition of excess known amount of sertaconazole to each screw capped test tube or vial sealed with rubber closure containing $2 \mathrm{ml}$ of liquid excipients. After sealing, the test tubes or vials were shaken in an isothermal shaker at $37 \pm 1^{0} \mathrm{C}$ for $72 \mathrm{~h}$. After 72 $\mathrm{h}$, each tube was centrifuged at 5000rpm using Remi centrifuge for $30 \mathrm{~min}$ and $0.1 \mathrm{ml}$ clear supernatant liquid was drawn and diluted suitably and analyzed by UV Visible spectrophotometry [6]. The selection criteria for surfactants, cosurfactants and organic solvents were the maximum solubility of drug in to given liquid and feasibility of respective component into topical formulation.

2.3 Research and development study

The literature is available on the selection of type and concentration of lipid, surfactant and other excipients used for the formulation of solid lipid nanoparticles. But actually the excipients used and their quantities were based on the method of preparations, the expected layout of lipid matrix formed, stability and compatibility of formulation. Hence there is a need to study the influence of formulation constituents and process parameters such as stirring rate, sonication time and heating temperature on SLN for preparation of successful formulation. The SERT-SLNs were formulated using modified solvent emulsification technique, the study parameters were varied while other constraints were kept constant.

2.4 Influence of process ingredients

2.4.1 Influence of lipid concentration [7]

As observed by lipid solubilizing potential the drug showed maximum solubility in two lipids glyceryl monostearate and OLML. The three formulations A1, A2 and A3 were prepared composing Drug: Lipid ratio 
1:5. The A1 formulation composed of GMS as lipid, A2 formulation composed of pre-lipid OLML and A3 formulation composed of mixture of GMS and OLML 1:1. The formulated SLN were evaluated for average particle size (APS) and polydispersity index (PDI).

2.4.2 Influence of surfactant concentration [8]

Surfactant OLMS has shown maximum amount of drug dissolution. The B1, B2 and B3 formulations were prepared with different OLMS concentrations as $2 \% \mathrm{v} / \mathrm{v}, 4 \% \mathrm{v} / \mathrm{v}$ and $6 \% \mathrm{v} / \mathrm{v}$ respectively and checked against the particle size and PDI

2. 4.3 Influence of organic solvent [9]

Organic solvents have shown the significant effect on the particle size, PDI and entrapment efficiency of SLN. The different formulations of $\mathrm{C} 1, \mathrm{C} 2, \mathrm{C} 3$ and $\mathrm{C} 4$ were formulated using dimethyl sulfoxide (DMSO) $1.5 \% \mathrm{v} / \mathrm{v}$, DMSO $5 \% \mathrm{v} / \mathrm{v}$, Acetone $1.5 \% \mathrm{v} / \mathrm{v}$ and Acetone $5 \% \mathrm{v} / \mathrm{v}$ respectively. The prepared SLNs were evaluated for APS and PDI.

2.4.4 Influence of Poloxamer 188 and Poloxamer 407 [10]

To achieve a maximum stability and induce a charge on solid lipid nanoparticles poloxamer 188 and poloxamer 407 (D1 and D2) were used in the formulation. The concentration of both poloxamers was kept as $0.5 \%$ w $/ \mathrm{v}$. The effect of poloxamers on SLN was checked by verifying APS, PDI and zeta potential (ZP) of formulation.

2.4.5 Influence of d-alpha tocopheryl polyethylene glycol 1000 succinate (TPGS)[11]

The concentration of TPGS used as solubilizing agent or as emulsifier is up to $2 \% \mathrm{w} / \mathrm{v}$. The TPGS have excellent solubilizing potential for the drug. The $0.05 \%, 0.5 \%, 1.5 \%, 3.5 \%$ and $5 \% \mathrm{w} / \mathrm{v}$ of TPGS were taken as $\mathrm{E} 1, \mathrm{E} 2, \mathrm{E} 3, \mathrm{E} 4$ and E5 to determine the coemulsifying potential.

2.4.6 Influence of stearic stabilizer [12]

A stearic stabilizer stabilizes the SLN for forming adsorptive layer around particle and inducing a charge on particle. Thereby it repels the approaching particles and maintains them in suspended form. Stearyl amine is an example of this class which is taken as $0.1 \% \mathrm{w} / \mathrm{v}(\mathrm{F} 1), 0.2 \% \mathrm{w} / \mathrm{v}(\mathrm{F} 2)$ and $0.3 \% \mathrm{w} / \mathrm{v}(\mathrm{F} 3)$ for formulation of SLN and evaluated for particle size, PDI and zeta potential.

2.5 Influence of process parameters

2.5.1 Influence of stirring rate [13]

Stirring rate and stirring time was optimized for the particle size and PDI. The stirring rate of $2000 \mathrm{rpm}, 3000$ $\mathrm{rpm}$ and $4000 \mathrm{rpm}$ (G1, G2 and G3) for $1 \mathrm{~h}$ was maintained and then APS and PDI were observed.

2.5.2 Influence of stirring time [14]

Stirring time was optimized for $1 \mathrm{hr}, 1.5 \mathrm{hr}$ and $2 \mathrm{hr}(\mathrm{H} 1, \mathrm{H} 2$ and $\mathrm{H} 3)$. The formulations were evaluated for APS and PDI.

2.5.3 Influence of sonication time [15]

The variation in sonication time from $0 \mathrm{~min}$ (I1), $10 \mathrm{~min}$ (I2), $20 \mathrm{~min}$ (I3) and $30 \mathrm{~min}$ (I4) have an influence in formulating SERT-SLN which was evaluated on the ground of APS, PDI and ZP.

2.5.4 Influence of cooling (Heating slow cooling and Heating sudden cooling) [15]

The formulated hot emulsion of SLN was cooled by two approaches J1 with slow cooling and J2 fast and sudden cooling at the time of stirring. The obtained formulations were studied for particle size and PDI.

2.6 Formulation of SERT-SLN

SLNs were prepared by a simple and modified solvent emulsification technique. On the basis of solubility study and pre-optimization study, the various variables at different concentrations and parameters were selected. After selecting the desirable constituents and process conditions, the SERT loaded SLNs were prepared. In brief, the drug was dissolved in DMSO, mixed in stearyl amine and lipid OLML (Natural stearin fraction of olive) and heated at $80^{\circ} \mathrm{C}$. TPGS and surfactant OLMS were dissolved in water and the mixture was heated at same temperature as lipid phase. Lipid phase was added into aqueous phase under constant stirring. This suspension was subjected to sudden decrease in temperature and the stirring was continued for $1 \mathrm{~h}$ at 2000 $\mathrm{rpm}$. The utility of this method is simple fabrication process and can be reproduced in lab scale.

2.7 Evaluation of SERT-SLN

2.7.1 Particle size and polydispersity index (PS and PDI)

The particle size and polydispersity index of SERT-SLN were determined by photon correlation spectroscopy (PCS) using a Zetasizer (Nano ZS 90, Malvern Instruments, UK) at 200C, by 900 scattering optics. The SLN dispersions were diluted with double distilled water with the concentration of 0.001 to $1 \%$ mass.

\subsubsection{Zeta Potential (ZP)}

The zeta potential measurement is taken as stability indicating tool for the SLN. The SLN dispersion samples were first diluted with double distilled water and then the ZP was determined by using Zetasizer (Nano ZS 90, Malvern Instruments, UK). ZP measurements were run at $25^{\circ} \mathrm{C}$ with electric field strength of $23 \mathrm{~V} / \mathrm{m}$.

2.7.3 Entrapment efficiency (EE) [16] 
The ability of lipid matrix to encapsulate the drug was determined by calculating the entrapment efficiency and drug loading. The cooling ultracentrifuge (Remi Instruments Ltd., Mumbai, India) was used to separate aqueous dispersion of SLN. The centrifugation was done at $60000 \mathrm{rpm}$ for $45 \mathrm{~min}$ at $4^{0} \mathrm{C}$ by placing $1.5 \mathrm{ml}$ of SERT-SLN dispersion into eppendorf tubes. After centrifugation, the dispersion separates in to supernatant clear aqueous phase and precipitated pellets of SERT-SLN. The concentration of SERT in the aqueous phase was determined using UV-visible spectrophotometer (UV 1700, Shimadzu, Japan) at $\lambda$ max 260 $\mathrm{nm}$. Values of EE \% and DL \% were calculated using Eqs. (1) and (2) respectively.

$$
\begin{aligned}
& \% \mathrm{EE}=\frac{\text { Total mass of SERT }- \text { Total mass of SERT in supernatant }}{\text { Total mass of drug }} \times 100 \\
& \% \mathrm{DL}=\frac{\text { Total mass of SERT }- \text { Total mass of SERT in supernatant }}{\text { Total mass of lipid }} \times 100
\end{aligned}
$$

2.7.4Transmission Electron Microscopy (TEM)

The particle size and PDI of SERT-SLN dispersion was confirmed by TEM analysis. Briefly, SERTSLN dispersion was prepared in water and One drop of SERT-SLN dispersion was placed on the copper grid coated with a thin film of carbon and dried for $45 \mathrm{~min}$ and then analyzed by transmission electron microscope Philips CM200 (Philips, Netherlands).

2.7.5 Differential scanning calorimetry (DSC) analysis

Differential scanning calorimeter (DSC 1 STARe System, Mettler-Toledo, Switzerland) was used to determine the thermal behavior of SERT, lipid and SERT-SLN. DSC scans were recorded at heating rate of $100 \mathrm{C} / \mathrm{min}$ in the range of $30-300^{\circ} \mathrm{C}$ and an empty standard aluminium pan was used as reference.

2.7.6 X-Ray diffraction study (XRD)

X-ray scattering measurements were carried out with a Philips PAN analytical expert PRO X-ray diffractometer 1780 to analyze the crystal morphology of SERT in SLN's. A Cu Ka radiation source was used, and the scanning rate $(2 \mathrm{~h} / \mathrm{min})$ was $50 \mathrm{C} / \mathrm{min}$. X-ray diffraction measurements were carried out on pure OLML, SERT and SERT-SLN.

\subsubsection{Stability Studies [17]}

The stability studies was done as per the $\mathrm{ICH}$ guidelines at four different storage conditions i.e refrigeration, $25 \pm 2{ }^{\circ} \mathrm{C} / 60 \% \mathrm{RH}, 30 \pm 2^{\circ} \mathrm{C} / 65 \% \mathrm{RH} \pm 5 \% \mathrm{RH}$ and $40 \pm 2{ }^{\circ} \mathrm{C} / 75 \% \mathrm{RH} \pm 5 \% \mathrm{RH}$. The samples of SERT-SLNs were withdrawn at the end of 1,2, 3 and 6 months and evaluated for physical appearance, clarity, particle size, PDI and entrapment efficiency.

2.7.8 Drug release study [18]

The different process variables and conditions also showed influence on the precipitation and formation of drug-lipid matrix which ultimately affects the release of drug from SLN. In vitro drug release study was performed using modified franz diffusion cell to study the release pattern of SERT-SLN compared with reference cream Ertaczo ${ }^{\circledR}$. Cellulose acetate membrane $(0.1 \mu \mathrm{m}$ pore diameter $)$ was used for diffusion study which was previously soaked with isopropyl myristate for $24 \mathrm{~h}$ to simulate the lipophilic properties of stratum corneum. The donar compartment was placed with weighed quantity of SERT-SLN gel and reference cream and phosphate buffer saline $\mathrm{pH} 7.2$ on receiver compartment. Sampling was done at predetermined rate over the period of $24 \mathrm{~h}$ and analyzed by UV spectrophotometer at $260 \mathrm{~nm}$.

\section{RESULT}

3.1 Preformulation study

The formulation of solid lipid nanoparticles commences with the selection process of excipients such as solid lipid, surfactant and others.

3.1.1 Selection of lipid

The selection of lipids has great influence on the particle size, pattern of drug loading and drug release. Broad variety of solid lipids were screened for formulation like stearic acid (SA), glyceryl monostearate (GMS), Myrj 49, compritol 888ATO, Lipid OLML and gelucire 44/14 along with kokum butter and mango butter to explore the use of natural excipients for topical use. Among different lipids stearic acid, GMS, gelucire and OLML exhibited the maximum solubilising property of drug sertaconazole at minimum concentration. The final lipid was selected on the basis of amount of lipid required to keep drug: lipid ratio at lower concentration which leads to smaller particle size. The lipids GMS and OLML with self emulsifying property and saturated C16-C22 chain length were selected assuming these solely or in combination will entrap more amount of drug and will have uniform small nanometric particle size [19]

3.1.2 Selection of surfactant 
Formation of spherical nanoparticles, increased entrapment and the stability of SLNs were influenced by the surfactant, cosurfactant and organic solvents. One or more surfactants were used in the formulation to study their influence on the stability of prepared formulations. Among different surfactant with varying hydrophile lipophile balance, cremophore RH 40, tween 80 and natural derived surfactant OLMS were found to be maximum solubilizing properties for the drug molecule. Cremophore induces allergic reaction when used for long term topical therapy hence was not selected. Tween 80 and OLMS both are reported to have low human toxicity. OLMS was selected as it is derived from natural excipient i. e fruit olive. It is more compatible with other ingredient and skin, safe and has more potential to repair the damaged skin conditions due to infection.

3.2 Formulation of SERT-SLN

With the insight of exploration of formulation of solid lipid nanoparticles at lab scale a preliminary study was conducted using solvent emulsification technique. A solvent emulsification technique was modified to obtain the uniform particle size and to get the desired properties of nanoparticles. The prepared SLNs were composed of drug, lipid, surfactant, cosurfactant/ organic solvents and water. The influence of concentration of lipid, surfactant, organic solvent, poloxamer, TPGS, stearic stabilizer and various process parameters on the formulation of SLN was studied. One variable was varied while others were kept constant. The other components such as organic solvent, poloxamer and stirring rate were used to improve the physicochemical properties of SLN

3.3 Influence process ingredients

3.3.1 Influence of lipid concentration

GMS and OLML showed maximum solubility of drug hence selected for the formulation of SLN. Three different formulations with only GMS (A1), only OLML (A2) and combination of two lipids GMS: OLML were prepared. The combinations of two lipids provide more space of imperfections which entraps more amount of drug inside the lipid matrix [20]. The particle size and PDI for the A3 formulation was found to be $293 \mathrm{~nm}$ and 0.5 respectively. The formulation A1 showed precipitation of GMS due to its low melting point 58$59^{\circ} \mathrm{C}$. The A2 formulation with OLML as lipid showed $213.3 \mathrm{~nm}$ particle size and 0.395 PDI (Fig 1A). Lipid OLML was selected for the further study and was kept constant in the ratio of 1:5 with drug.

3.3.2 Influence of surfactant concentration

The effective concentration of surfactant is required to provide stability and narrow size distribution of nanoparticles. Excess use of surfactant can interfere with loading properties of SLN [21]. As the concentration of OLMS increases from 2, 4 and $6 \% \mathrm{w} / \mathrm{v}$ the particle size first decreases but remains stable for further rise in surfactant concentration. The particle size and PDI were noticed to be $318.9 \mathrm{~nm}$ with 0.423 for $2 \%$ w/v surfactant while increase in surfactant concentration from $2 \%$ to $4 \%$ decreases the particle size from $318.9 \mathrm{~nm}$ to $201 \mathrm{~nm}$ with PDI 0.269. Further increase in surfactant concentration $6 \% \mathrm{w} / \mathrm{v}$ does not cause decrease in particle size which was found to be $186.3 \mathrm{~nm}$ and 0.328 PDI (Fig 1B).

3.3.3 Influence of organic solvent

With purpose to improve the solubility of drug in to lipid, entrapment of drug inside lipid and formulate the nanoparticles with uniform particle size distribution various organic solvents were screened and few were found to be efficient to reduce the particle size. IIg limit for DMSO and acetone for topical route is $45.5 \%$ and 12.5 - $45 \%$ respectively which can be used without any toxicity [22]. The APS and PDI of C1 formulation were found to be $237.6 \mathrm{~nm}$ and 0.375 whereas for $\mathrm{C} 3$ formulation APS and PDI were found to be $281.9 \mathrm{~nm}$ and PDI 0.531 (Particle analysis study report: poor data quality due to too poly dispersed sample for cumulative data analysis). The C2 sample showed APS $205.7 \mathrm{~nm}$ and PDI 0.349 as shown in table 1 . Polydispersity index was found to be on higher side i. e. up to 1 for the $\mathrm{C} 4$ formulation hence it was rejected from the study. (Fig 1C)

Table 1. Influence of organic solvent concentration on APS and PDI

\begin{tabular}{|l|l|l|ll|ll|ll|l|}
\hline Formulations & PDI & $\begin{array}{l}\text { Average } \\
\text { Particle Size } \\
(\mathrm{nm})\end{array}$ & $\begin{array}{l}\text { Peak 1 \% } \\
\text { intensity }\end{array}$ & $\begin{array}{l}\text { Peak 2 \% } \\
\text { intensity }\end{array}$ & $\begin{array}{l}\text { Peak 3 \% } \\
\text { intensity }\end{array}$ \\
\hline C1 & 0.375 & 237.6 & - & & - & & - & \\
\hline C2 & 0.349 & 205.7 & $\begin{array}{l}206.1 \\
86 \%\end{array}$ & $\mathrm{~nm}$ & $\begin{array}{l}1010 \\
14 \%\end{array}$ & $\mathrm{~nm}$ & $\begin{array}{l}4816 \\
5.7 \%\end{array}$ & $\mathrm{~nm}$ \\
\hline C3 & 0.531 & 281.9 & & & & & & \\
\hline C4 & 1 & - & & & & & \\
\hline
\end{tabular}

3.3.4 Influence of Poloxamer 188 and Poloxamer 407

Poloxamer 188 and poloxamer 407 are reported to induce strong ionic charges to the SLN. The APS and PDI were observed to be $237.7 \mathrm{~nm}$ and 0.422 for poloxamer 188 and $302 \mathrm{~nm}$ and 0.530 for poloxamer 407 (Table 2). The zeta potential was found to be near to $-20.5 \mathrm{mV}$ for both the poloxamers (Fig 1D). 
Table 2. Influence of poloxamer 188 and poloxamer 407concentration on APS, PDI and zeta potential

\begin{tabular}{|l|l|l|ll|ll|ll|l|}
\hline Formulations & PDI & $\begin{array}{l}\text { Average } \\
\text { Particle } \\
\text { Size (nm) }\end{array}$ & $\begin{array}{l}\text { Peak 1 \% } \\
\text { intensity }\end{array}$ & $\begin{array}{l}\text { Peak 2 \% } \\
\text { intensity }\end{array}$ & $\begin{array}{l}\text { Peak 3 \% } \\
\text { intensity }\end{array}$ & $\begin{array}{l}\text { Zeta } \\
\text { Potential } \\
\mathrm{mV}\end{array}$ \\
\hline D1 & 0.422 & $237.7 \mathrm{~nm}$ & & & & & -20.5 \\
\hline D2 & 0.530 & $302 \mathrm{~nm}$ & $\begin{array}{l}477.7 \\
51.2 \%\end{array}$ & $\mathrm{~nm}$ & $\begin{array}{l}161.5 \\
47.3 \%\end{array}$ & $\mathrm{~nm}$ & $\begin{array}{l}5560 \\
1.5 \%\end{array}$ & $\mathrm{~nm}$ & -19.8 \\
\hline
\end{tabular}

3.3.5 Influence of d-alpha tocopheryl polyethylene glycol 1000 succinate (TPGS)

TPGS has been used as solubilizing agent, coemulsifier, occlusive and emollient agent. Here, attempt has been made to determine concentration of TPGS effective to reduce the particle size in association with OLMS. The particle size was found to be $201 \mathrm{~nm}$ for E1, $231 \mathrm{~nm}$ for E2, $133.3 \mathrm{~nm}$ for E3, $179.8 \mathrm{~nm}$ for E4 and $178.8 \mathrm{~nm}$ for E5 as shown in table 3. The PDI for the given formulations were 0.485 for E1, 0.458 for E2, 0.381 for E3, 0.425 for E4 and 0.481 for E5 (Fig 1E). The E3 formulation with 1.5\% w/v of TPGS was observed to be the most significant having smallest particle size and monodispersed particles.

Table 3. Influence of TPGS concentration on APS and PDI

\begin{tabular}{|c|c|c|c|c|c|}
\hline Formulations & PDI & $\begin{array}{l}\text { Average } \\
\text { Particle Size } \\
(\mathrm{nm})\end{array}$ & $\begin{array}{l}\text { Peak 1 \% } \\
\text { intensity }\end{array}$ & $\begin{array}{l}\text { Peak } 2 \% \\
\text { intensity }\end{array}$ & $\begin{array}{ll}\text { Peak } 3 & \% \\
\text { intensity } & \end{array}$ \\
\hline E1 & 0.485 & $201 \mathrm{~nm}$ & $\begin{array}{ll}335.5 & \mathrm{~nm} \\
49.2 \% & \end{array}$ & $\begin{array}{ll}125.3 & \mathrm{~nm} \\
46.3 \% & \end{array}$ & $\begin{array}{ll}5208 & \mathrm{~nm} \\
4.5 \% & \end{array}$ \\
\hline E2 & 0.458 & $231 \mathrm{~nm}$ & $\begin{array}{ll}128.7 & \mathrm{~nm} \\
90.9 & \\
\end{array}$ & $\begin{array}{ll}148.98 & \mathrm{~nm} \\
9.1 \% & \end{array}$ & \\
\hline E3 & 0.381 & $133.3 \mathrm{~nm}$ & $101.4 \mathrm{~nm}$ & & \\
\hline E4 & 0.425 & $179.8 \mathrm{~nm}$ & $\begin{array}{l}163.6 \\
75.9 \\
\end{array}$ & $\begin{array}{l}902.7 \\
22.4 \% \\
\end{array}$ & $\begin{array}{l}5152 \\
3.2 \%\end{array}$ \\
\hline E5 & 0.481 & $178.8 \mathrm{~nm}$ & $\begin{array}{l}148.3 \\
75.9\end{array}$ & $\begin{array}{ll}590.2 & \mathrm{~nm} \\
21.7 \% & \\
\end{array}$ & $\begin{array}{l}5369 \\
2.4 \%\end{array}$ \\
\hline
\end{tabular}

3.3.6 Influence of stearic stabilizer

The stearic stabilizer used in this study is stearyl amine. Along with charge inducer properties, stearyl amine has lipoidal characteristic hence it can be responsible for the formation of larger or polydispersed nanoparticles [23]. APS, PDI and zeta potential for different concentration of stearyl amine $0.1 \%(\mathrm{~F} 1), 0.2 \%$ (F2) and $0.3 \%$ (F3) on the formulation of SLN were found to be $257.3 \mathrm{~nm}$ APS, 0.526 PDI and $-9.42 \mathrm{mV}$ ZP for F1, $253.7 \mathrm{~nm}$ APS, 0.449 PDI and 11.4 mV ZP for F2 and 212.1 nm APS, 0.299 PDI and +26.4 mV ZP for F3 (Fig 1F). 


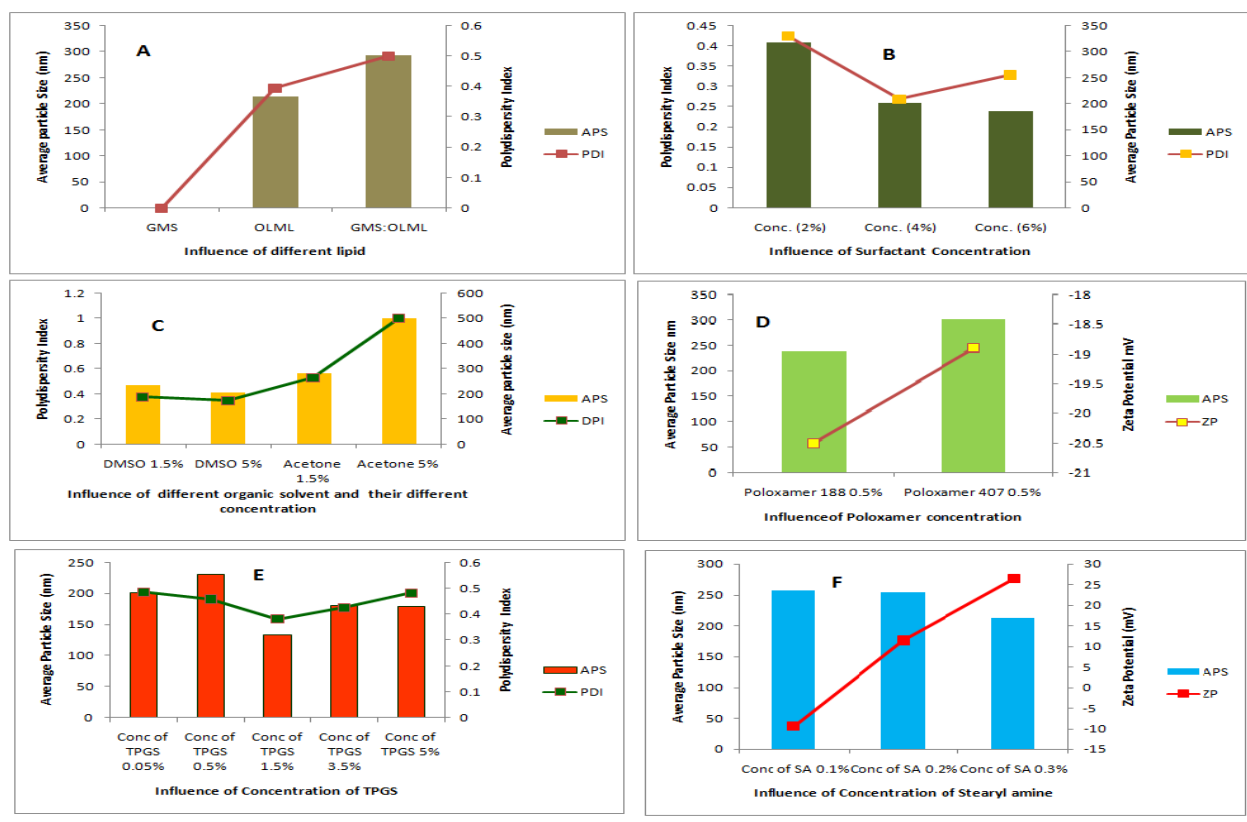

Fig. 1. Graph of influence of formulation ingredients on average particle size, polydispersity index and zeta potential (A) Influence of different lipid, (B) Influence of surfactant concentration, (C) Influence of different organic solvent and their different concentration, (D, Influence of poloxamer concentration, (E) Influence of concentration of TPGS and (F) Influence of stearyl amine concentrations on APS, PDI and ZP.

3.4 Influence of process parameters

3.4.1 Influence of stirring rate

To obtain the smaller particle size with narrow particle size distribution the stirring rates were optimized. The formulation having $2000 \mathrm{rpm}$ stirring rate has $227.4 \mathrm{~nm}$ APS and 0.293 PDI, stirring rate of $3000 \mathrm{rpm}$ has $229 \mathrm{~nm}$ APS and 0.278 PDI and for $4000 \mathrm{rpm}$ has $245 \mathrm{~nm}$ APS and 0.417 PDI (Fig 2G). The increase in stirring rate has not shown much significant effect on lowering the particle size and polydispersity index. Hence the formulation G1 was selected for further study.

3.4.2 Influence of stirring time

The stirring period of $1 \mathrm{hr}, 1.5 \mathrm{hr}$ and $2 \mathrm{hr}$ were used to prepare solid lipid nanoparticles. The APS and PDI of formulation H1, H2 and H3 were found to be $219 \mathrm{~nm}$ and $0.271,223 \mathrm{~nm}$ and 0.298 and $269 \mathrm{~nm}$ and 0.362 (Fig $2 \mathrm{H}$ ) respectively. Greater time of stirring increases the particle size and polydispersity index. Hence the formulation with stirring time $1 \mathrm{hr}$ and stirring rate $2000 \mathrm{rpm}$ was selected.

3.4.3 Influence of sonication time

The APS and PDI for I1 formulation were found to be $231.9 \mathrm{~nm}$ and 0.289 , for I2 formulation $240 \mathrm{~nm}$ and 0.376 , for I3 formulation $254.5 \mathrm{~nm}$ and 0.393 and for I4 formulation $285 \mathrm{~nm}$ and 0.472 (Fig 2I) respectively. Surprisingly, sonication showed increase in particle size with increase in sonication time. The possible explanation for this is coagulation of nanometric particles when they collide with each other due to sonic waves [24].

3.4.4 Influence of temperature (Heating slow cooling and Heating sudden cooling)

Temperature is a major factor which decides the precipitation of lipid and drug from emulsion and the formation of type of lipid polymorphs. With reference to the fact that the lipid polymorphs existing in $\alpha$ and $\beta$, form have maximum capacity to entrap drug molecule and also exist in amorphous nature, here attempt has been made to formulate thermodynamically unstable lipid structure by sudden cooling of emulsion at the time of stirring [25]. Two approaches were studied - one with slow cooling of emulsion at the time of stirring and second approach fast and sudden cooling of emulsion. The APS and PDI of both the formulations were found to be $279 \mathrm{~nm}$ and 0.445 for $\mathrm{J} 1$ formulation and $229 \mathrm{~nm}$ and 0.304 for J2 formulation (Fig 2J) respectively. J2 formulation with sudden cooling causes sudden solidifying nanoparticles and causes no longer collision and agglomeration. 


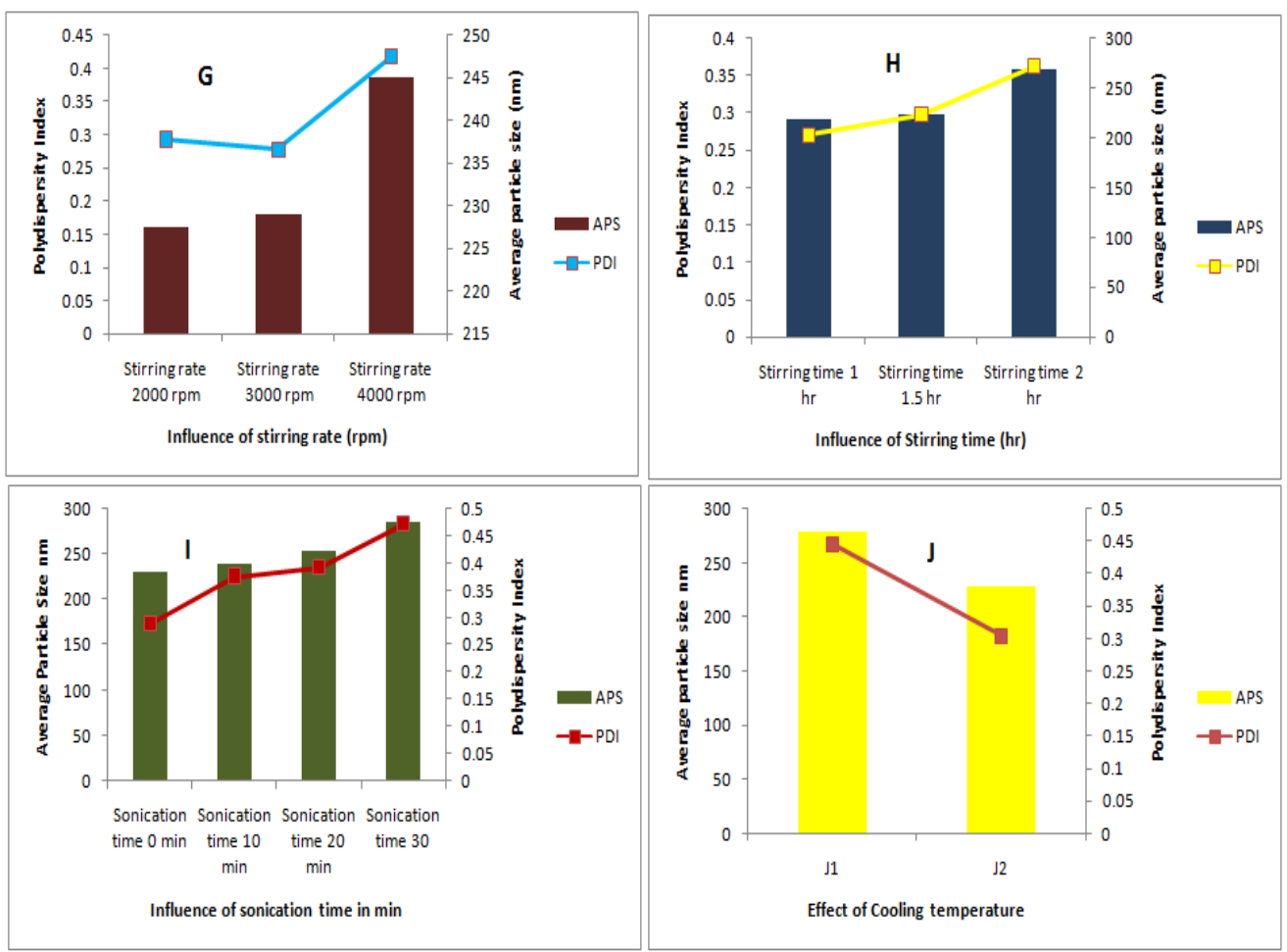

Fig. 2. Graph of influence of process parameters on average particle size and zeta potential. (G)Influence of Stirring rate, (H) Influence of stirring time, (I) Influence of sonication time, (J) Effect of cooling temperature on APS and PDI.

3.5 Evaluation of SERT-SLN

The SERT-SLN was formulated with different optimized parameters based on the above studies. Minimum particle size, minimum polydispersity index and higher zeta potential were basic criteria for obtaining optimum solid lipid nanoparticles. As discussed earlier, SLN were prepared by a simple and modified solvent emulsification technique. On the basis of solubility study and pre-optimization study, various variables at different levels were selected. The drug was dissolved in DMSO, mixed in stearyl amine and lipid OLML (Natural stearin fraction of olive) and heated at $80^{\circ} \mathrm{C}$. TPGS and surfactant OLMS were dissolved in water which was heated at same temperature as lipid phase. Lipid phase was added into aqueous phase under constant stirring. The stirring was continued in ice bath to obtain sudden decrease in temperature and the stirring was continued for $1 \mathrm{hr}$ at $2000 \mathrm{rpm}$.

3.5.1 Particle size, PDI and Zeta Potential

Particle size and PDI of SERT-SLN dispersion prepared using above method was found to be $155.1 \mathrm{~nm}$ and 0.23 respectively. The zeta potential of optimized batch was observed to be $9.76 \mathrm{mV}$.

3.5.2 Entrapment efficiency

The EE and DL of optimized formulation were found to be $93 \%$ and $15.4 \%$ respectively.

3.5.3 Transmission Electron Microscopy (TEM)

The particle size analysis TEM image of SERT-SLN reveals that nanoparticles are spherical in shape and confirmed to be near to $200 \mathrm{~nm}$.

3.5.3 DSC

The DSC thermograms of pure drug sertaconazole, pure lipid OLML and SERT-SLN are shown in figure 3. Thermograms of Pure drug sertaconazole and lipid OLML exhibited endothermic peak at $156.98^{\circ} \mathrm{C}$ and $63.20^{\circ} \mathrm{C}$ respectively revealing its melting and existence of crystalline nature. The peak for sertaconazole is completely absent in SERT-SLN thermogram while a sharp peak for OLML $48.35^{\circ} \mathrm{Cchanged}$ to small and broad peak which is shifted to left side suggesting configuration of SLN in amorphous form with many imperfections in which amorphous drug is entrapped. This particular behavior of SLN is due to change in the formulation process. Sudden cooling of SLN causes the formation of $\alpha$ state of lipid with many lattice defects which is exact place where drug, surfactant or organic phase can be entrapped. 


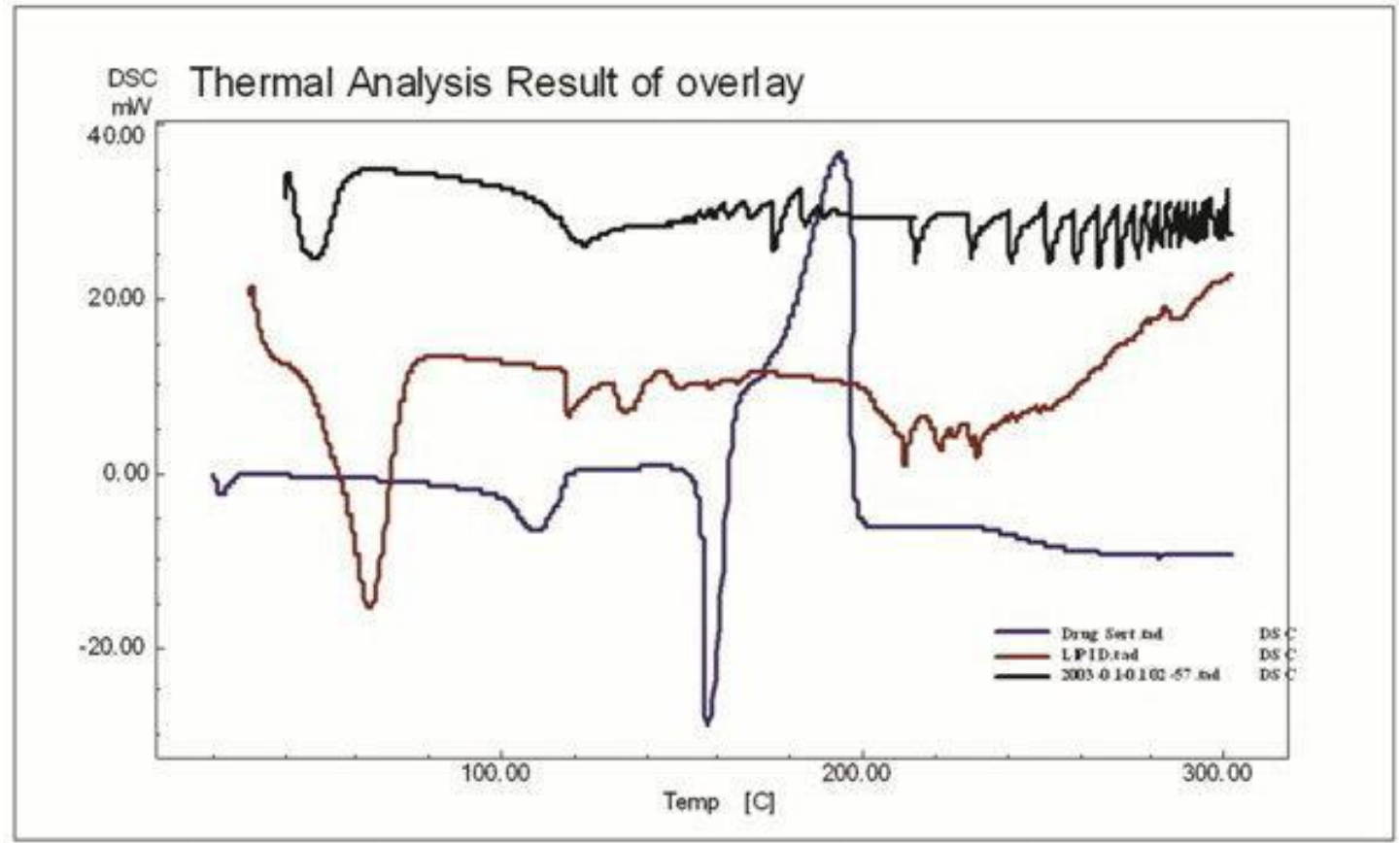

Fig. 3. DSC thermogram of SERT-SLN (A), pure lipid OLML (B) and drug sertaconazole. 3.5.4 XRD

The X-ray diffraction patterns of pure drug SERT (a), SERT-SLN (b) and lipid OLML (c) are shown in figure 4. Pure drug sertaconazole and pure lipid OLML showed a sharp typical crystalline peaks pattern revealing presence of crystalline nature of both. While XRD pattern for SERT-SLN showed no intrinsic peaks, all the major characteristic peaks for sertaconazole and OLML were absent in SERT-SLN, only similar broad and small peaks were observed, showing sertaconazole and OLML were present in amorphous state in SERTSLN.
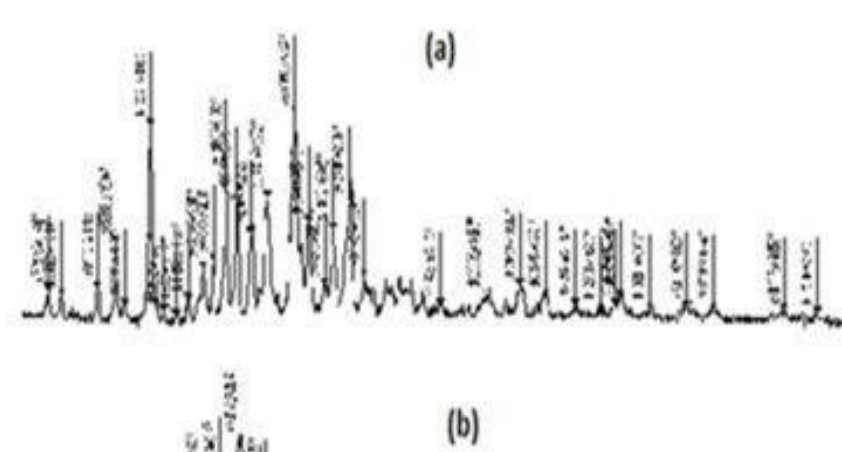

(b)

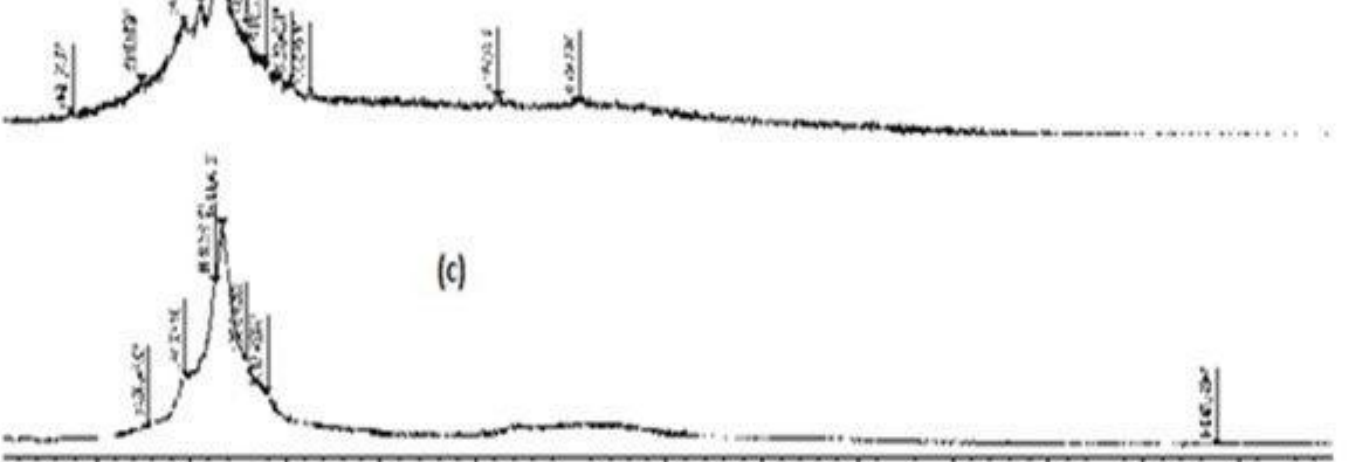

Figure 4. Comparison of $\mathrm{X}$ ray diffraction pattern: sertaconazole, lipid OLML and SERTSLN. 


\subsubsection{Stability Study}

After stability storage period of 6 months at four different temperatures all the samples were found to be stable and opaque. Refrigerated samples were found to be most stable and unchanged. The slight increase in particle size, increase in PDI and decrease in entrapment efficiency with increase in temperature was observed for the samples stored at higher temperature. Figure 3 reveals the results of APS, PDI and ZP at different storage conditions after stability period.

3.5.6 Drug release study

The drug release study of SERT-SLN through porcine skin was studied and it was found that the rate of permeation of sertaconazole was significantly higher in case of SLN formulation as compared to reference. The plot of cumulative amount of drug permeated showed 5.42 times higher drug release rate when compared with reference cream. The flux value calculated from the linear portion of graph Q/A vs time (figure 5) from SLN gel was found to be $7.5 \pm 0.392 \mathrm{mcg} / \mathrm{cm}^{2} \mathrm{~h}$ and $4 \pm 0.198 \mathrm{mcg} / \mathrm{cm}^{2} \mathrm{~h}$ for reference cream.

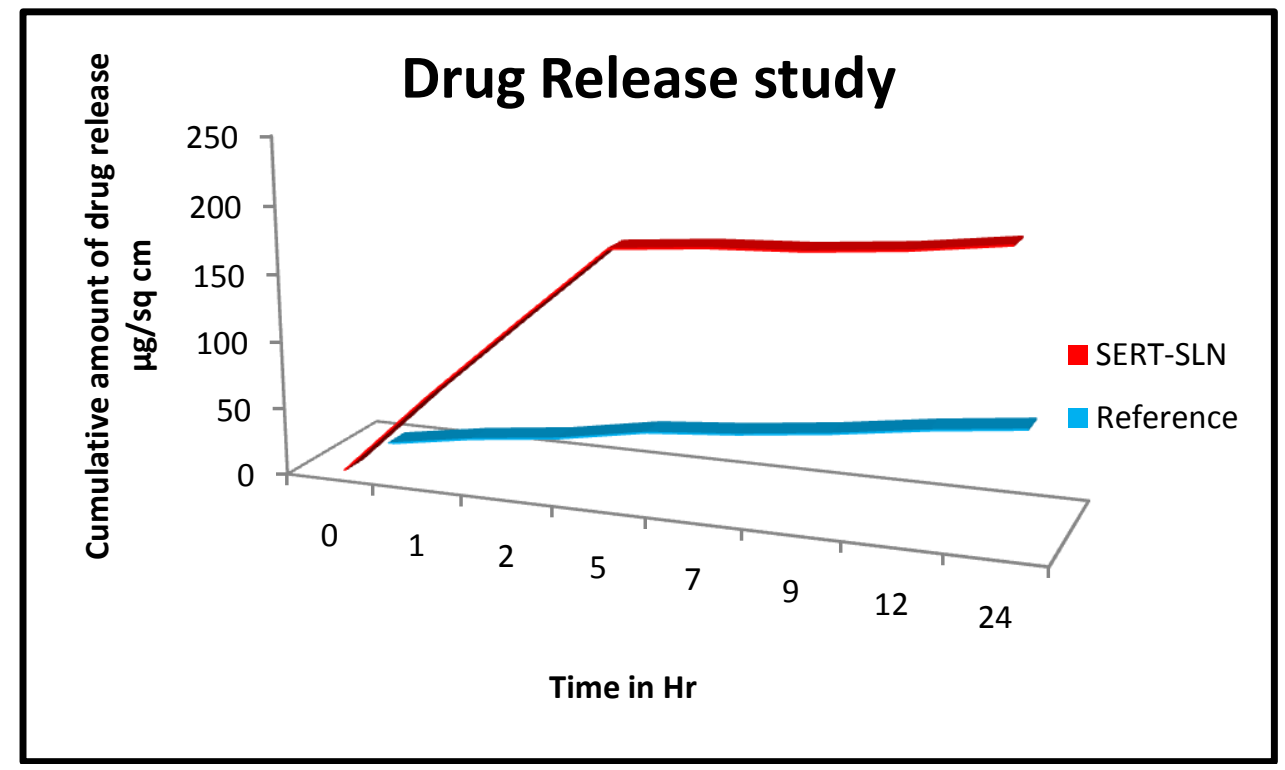

Figure 5. In vitro skin permeation profile of SERT from SLN gel and reference marketed cream.

\section{DISCUSSION}

The SERT-SLN were formulated using solid lipid GMS and OLML which are 2,3-dihydroxypropyl octadeconoate with $\mathrm{C} 21$ carbon chain length and mixture of cetearyl and sorbitan of olive fruit derivative with self emulsifying property and saturated C16-C22 carbon chain length respectively. This makes a perfect component for loading of drug into solid lipid matrix. The nonionic surfactant OLMS is selected which has emollient, solubilizing and coemulsifying properties. It is water soluble and has ability to recover sebum from skin. It helps drug to partition in lipid phase and gives maximum entrapment. The other excipients such as poloxamers for imparting charge to particle, TPGS for aiding solubilizing of drug in solid lipid, stearyl amine for inducing positive charge to particles and DMSO as solubilizing agent help to form nanodroplets. The entrapment of DMSO into lipid matrix improves physicochemical properties of SLN. Modified solvent emulsification method is implemented with the use of organic solvent DMSO (IIg limit upto 45.5\%v/v for topical drug delivery). DMSO also works as solubilizing agent and penetration enhancer hence shown to improve skin targeting [26]. DMSO acts by two ways - one by solubilizing the drug as well as lipid and other after solidification process as nanodroplet containing drug entrapped inside imperfect crystal lattice which precipitates in SLN and behaves as drug reservoir. DMSO being a good penetration enhancer helps to penetrate SLN in to deeper skin strata. Low and very high concentrations of DMSO in SLN give polydisperse nanoparticles. Low concentration of DMSO was unable to dissolve lipid and drug while the higher concentration of DMSO causes extra DMSO nanodroplets which are not entrapped inside lipid matrix hence improper precipitation of drug, lipid and other excipients occurs. Hence ultimately particle size and polydispersity increases.Nanometric particles with uniform particle size can be obtained with modified solvent emulsification method. The type of lipid and its concentration have great influence on the SLN. Lipid OLML have smaller nanometric particle size compared to GMS and OLML: GMS combination. This could be due to carbon chain length which has maximum solubilizing potential for drug molecule and hence impart smaller size. 
The increase in surfactant concentration decreases the particle size. This is due to the fact that higher surfactant concentration gives more area to cover for newly formed nanoparticles. The higher concentration of surfactant and higher temperature causes the drug to partition in between aqueous and lipid phase at the time of nanoemulsion formation but the solidification of lipid and drug from the emulsion occurs inappropriately which leads to polydispersed nanoparticles. Due to this reason 4\%v/v of OLMS is used in formulation.

TPGS is an effective solubilizing agent and coemulsifying agent which helps to achieve more uniform size nanoparticles [27]. It is a low melting solid hence get precipitated at the end of process of SLN formulation. Hypothetically TPGS may remain on the surface layer of SLN which may act as diffuse layer in which drug is partitioned and concentrated. When the formulation is applied on skin, TPGS with the help of DMSO accelerate the skin penetration. TPGS with $1.5 \%$ gives the best results for particle size and PDI. Higher and lower level causes either improper partitioning of drug or insufficient concentration of drug in lipid phase. Stearyl amine contains a lipophilic hydrocarbon chain which on adsorption onto particle projects its amine group towards aqueous phase which gives positive zeta potential. Skin carries negative charge hence targeting skin with the positively charged nanoparticles gives an opportunity to bind the SLN with the stratum corneum cells. Poloxamer 188 and poloxamer 407 have shown excellent negative zeta potential values but due to skin negative charges there may be a chance of repulsion of SLN particle from stratum corneum cell [3]. Hence for proper adhesion of SLN and for targeting to skin poloxamer was replaced with stearyl amine with the maximum concentration of $0.3 \% \mathrm{w} / \mathrm{v}$. Stearyl amine with $0.1 \% \mathrm{w} / \mathrm{v}$ and $0.2 \% \mathrm{w} / \mathrm{v}$ concentration were unable to induce sufficient positive charges to SLN.Higher stirring rate and time and sonication time causes the collision of nanoparticles and increase in particle size and polydispersity index. Collision causes formation of agglomerates of SLN and rise in particle size.The sudden change in temperature from hot nanoemulsion to cold nanodispersion causes sudden precipitation of drug, solid lipid, TPGS and stearyl amine. When the crystalline substance is melted and solidified, it again crystallizes in to three different polymorphic forms - unstable $\alpha$ form with maximum lattice defects and maximum entrapment efficiency, unstable $\beta$ ' form with some lattice defects and entrapment efficiency and most stable $\beta$ form with perfect crystal lattice and no lattice defects and entrapment of drug. By sudden cooling we tried to get $\alpha$ form and $\beta$ ' form with numerous lattice defects which gives amorphous property to our formulation and maximum entrapment efficiency.

Particle Size and polydispersity index of SERT-SLN sample were studied and the obtained results revealed that the SERT-SLN were appeared to be in nanometric size, with narrow particle size distribution which would be needed for deep skin penetration. The zeta potential value $9.76 \mathrm{mV}$ indicates the stability of SLN dispersion. In accordance to particle size analysis, TEM image of SERT-SLN shows the presence of spherical particle in the range of $150 \mathrm{~nm}$ to $300 \mathrm{~nm}$. The presence of DMSO and TPGS helps to improve entrapment of drug in lipid matrix.The DSC thermal analysis of SERT-SLN, Lipid OLML and pure drug SERT are given in figure 7. As shown by XRD study and DSC thermogram, pure drug SERT and lipid OLML were present as crystalline form while after the preparation of SERT-SLN the crystalline nature converts into amorphous nature. The amorphous SLN exists with lots of imperfections and more lattice defects incorporating more amount of drug.

Due to compatibility of lipid OLML, surfactant OLMS and stearyl amine, the SERT-SLN were found to be stable for longer period of time. Storage at refrigerated temperature shows more stable properties than the higher temperature. No significant increase in particle size, PDI and EE was observed over a period of 6 months at different temperature.

The permeation profile of SERT-SLN reveals initial burst release and then sustained release. The drug release for SERT-SLN was significantly higher compared to reference suggesting the formation of depot of drug in the skin strata.

\section{CONCLUSION}

The sertaconazole loaded lipid nanoparticles were optimized and prepared successfully by modified solvent emulsification technique with good entrapment efficiency and stability. The importance of formulation factors including type of lipid, concentration of surfactant, other ingredients and process variables such as stirring rate, stirring time effect of cooling temperature were provided by this analysis. Some of these factors affect particle size and polydispersity index while some factors have great influence on zeta potential. Use of OLML lipid, $4 \% \mathrm{v} / \mathrm{v}$ surfactant, $1.5 \% \mathrm{v} / \mathrm{v}$ DMSO and $1.5 \% \mathrm{w} / \mathrm{v}$ TPGS showed a significant impact on particle size and polydispersity index. Stearyl amine $0.3 \% \mathrm{w} / \mathrm{v}$ gave cationic charges to nanoparticles which are accepted for topical drug delivery. Process parameters also had great impact on particle size and size distribution of particles. Stirring rate of $2000 \mathrm{rpm}$ for $1 \mathrm{hr}$ and sudden cooling down the solution makes the formulation more uniform in size and found to have higher entrapment efficiency. From above optimized parameters the formulated SLN were characterized for TEM, DSC and XRD study and the nanoparticles were observed to be in spherical shape and size less than $200 \mathrm{~nm}$. The nanoparticles existed in an amorphous state. Release study reveals the biphasic release pattern which forms depot of drug in skin. In the conclusion, initial preformulation study and optimization of formulation parameters have clearly shown their usefulness in understanding SLN 
formation and this study provides a framework for further research in the area of topical drug delivery of solid lipid nanoparticles.

\section{ACKNOWLEDGEMENTS}

The authors are grateful to Dr. Nandurikar from Chem House Marketing, for supply of gift sample of lipid and surfactant and also for his guidance for fabrication of SLN. Further we extend our gratitude to Niksha Laboratories, Hyderabad for XRD study.

\section{REFERENCE}

[1] R. Muller, K. Mader And S. Gohla, Solid Lipid Nanoparticles (Sln) For Controlled Drug Delivery-A Review Of The State Of The Art, Eur J Pharm Biopharm, 50, 2000, 161-177.

[2] E. Bseiso, M. Nasr, O. Sammour And N. Gawad, Recent Adavances In Topical Formulation Carriers Of Antifungal Agents, Indian J Dermatology, Venereology And Leprology, 81 (5), 2015, 457-463.

[3] O. Uchechi, D. John, N. Ogbonna And A. A. Attama, Nanoparticles For Dermal And Transdermal Drug Delivery In, A Sezer (Ed), Nanotechnology And Nanomaterials "Application Of Nanotechnology In Drug Delivery" (Intech Publisher, 2014)

[4] J. Aqut, C. Palacin, J. Salqado, E. Casas, A. Sacristan And J. Ortiz, Direct Membrane -Damaging Effect Of Sertaconazole On Candida Albicans As A Mechanism Of Its Fungicidal Activity, Arzneimittelforschung 42 (5a,)1992, 721-724.

[5] K. Shah, A. Date, M. Joshi And V. Patravale, Solid Lipid Nanoparticles (Sln) Of Tretinoin: Potential In Topical Delivery, Int J Pharm, 345, 2007, 163-171.

[6] M. Joshi And V Patravale, Nanostructured Lipid Carrier (Nlc) Based Gel Of Celecoxib, Int J Pharm, 346, 2008, 124-132

[7] M. Rawat, A. Jain, S. Singh, Studies On Binary Lipid Matrix Based Solid Lipid Nanoparticles Of Repaglinide: In Vitro And In Vivo Evaluation, J Pharm Sci, 100(6), 2011, 2366-78.

[8] T. Helgason, T. Awad, K. Kristbergsson, D. Mcclements And J. Weiss, Effect Of Surfactant Surface Coverage On Formation Of Solid Lipid Nanoparticles (Sln), J Colloid Interface Sci, 334(1), 2009, 75-81.

[9] G. Cynthia, J. Swarnavalli, D. Roopsingh, V. Joseph And V. Kannappan, Role Of Solvents And Stabilizer In The Preparation Of Solid Lipid Nanoparticles Of Stearic Acid By Ultrasound Assisted Nanoprecipitation Method, Int J Innovative Research Science, Engineering And Technology, 4(1), 2015, 141-145.

[10] S. Honary And F Zahir, Effect Of Zeta Potential On The Properties Of Nano-Drug Delivery Systems-A Review (Part 1), Tropical J Pharm Research, 12(2), 2013, 255-264.

[11] S. Tan And N. Billa, Lipid Effects On Expulsion Rate Of Amphotericin B From Solid Lipid Nanoparticles, Aaps Pharmscitech, 15 (2), 2014, 287-95.

[12] E. Silva, G. Carneiro, D Araujo, J. Trindade, M. Yoshida, R. Orifice, M. Farias, M. Carvalho, S. Santos, G. Goulart, R. Alves And L. Ferreira, Solid Lipid Nanoparticles Loaded With Retinoic Acid And Lauric Acid Asan Alternative For Topical Treatment Of Acne Vulgaris, J Nanosci Nanotechnol, 15(1), 2015, 792-9.

[13] S. Mukherjee, S. Ray And R. Thakur, Solid Lipid Nanoparticles: A Modern Formulation Approach In Drug Delivery System, Indian J Pharm Sci, 71 (4), 2009, 349-58.

[14] M. Shah, Y. Agrawal, K. Garala And A. Ramkishan, Solid Lipid Nanoparticles Of A Water Soluble Drug Ciprofloxacin Hydrochloride, Indian J Pharm Sci, 74 (5), 2012, 434-42.

[15] A. Siddiqui, A. Alayoubi, Y. El-Malah And S. Nazzal, Modeling The Effect Of Sonication Parameters On Size And Dispersion Temperature Of Solid Lipid Nanoparticles (Slns) By Response Surface Methodology (Rsm), Pharm Dev Technol, 19(3), 2014, 342-6.

[16] H. Yang And M. Hon, The Effect Of The Degree Of Deacetylation Of Chitosan Nanoparticles And Its Characterization And Encapsulation Efficiency On Drug Delivery, Polym Plast Technol Eng, 49,2010, 1292-1296.

[17] S. Chalikwar, V. Belgamwar, V. Talele, S. Surana And M. Patil, Formulation And Evaluation Of Nimodipine-Loaded Solid Lipid Nanoparticles Delivered Via Lymphatic Transport System, Coll Surf B: Biointerf, 97, 2012, 109-116.

[18] V. Jenning, A. Thunemann And S. Gohla, Characterisation Of Novel Solid Lipid Nanoparticles Carrier System Based On Binary Mixtures Of Liquid And Solid Lipids, Int J Pharm, 199, 2000, 167-177.

[19] S. Labhade, P. Gide And R. Saudagar, Solid- Lipid Nanoparticles: A Newer Approach For Formulation And Optmization Of Hmg-Coa Reductase Inhibitors, Research J Pharma, Bio And Chem Sci, 4(3), 2013, 1297-1310.

[20] R. Bhandari And I. Kaur, A Method To Prepare Solid Lipid Nanoparticles With Improved Entrapment Efficiency Of Hydrophilic Drugs, Current Nanoscience, 9(2), 2013, 211-220. 
[21] M. Patel, S.Lakkadwala, M. Majrad, E. Injeti, S. Gollmer, Z. Shah, S. Boddu, And J. Nesamony, Characterization And Evaluation Of 5-Fluorouracil-Loaded Solid Lipid Nanoparticles Prepared Via A Temperature-Modulated Solidification Technique, Aaps Pharmscitech, 15(6), 2014, 1498-1508.

[22] Http://Www.Accessdata.Fda.Gov/Scripts/Cder/Iig/Getiigweb.Cfm (12/05/2016).

[23] C. Pardeshi, P. Rajput, V. Belgamwar, A. Tekade, G. Patil, K. Chaudhary And A. Sonje, Solid Lipid Based Nanocarriers: An Overview, Acta Pharm, 62, 2012, 433-472.

[24] N. Yadav, S. Khatak And U. Sara, Solid Lipid Nanoparticles- A Review, Int J Applied Pharma, 5(2), 2013,8-18.,

[25] T. Awad, T. Helgason, K. Kristbergsson, E. Decker, J. Weiss And D. Mcclements, Effect Of Cooling And Heating Rates On Polymorphic Transformations And Gelation Of Tripalmitin Solid Lipid Nanoparticle (Sln) Suspensions, Food Biophysics, 3(2), 2008, 155-162.

[26] I. Ozgüney, H. Karasulu, G. Kantarci, S. Sözer, T. Güneri, And G. Ertan, Transdermal Delivery Of Diclofenac Sodium Through Rat Skin From Various Formulations, Aaps Pharmscitech, 7(4), 2006, E39E45.

[27] T. Yuan, L. Qin, Z. Wang, J. Nie, Z. Guo, G. Li and C. Wuasian, Solid Lipid Dispersion Of Calcitriol With Enhanced Dissolution And Stability, J Pharm Sci, 8(1), 2013, 39-47 . 